\title{
Failure of Nehari's theorem for multiplicative Hankel forms in Schatten classes
}

Article

Accepted Version

Brevig, O. F. and Perfekt, K.-M. (2015) Failure of Nehari's theorem for multiplicative Hankel forms in Schatten classes. Studia Mathematica, 228 (2). pp. 101-108. ISSN 0039-3223 doi: https://doi.org/10.4064/sm228-2-1 Available at https://centaur.reading.ac.uk/71479/

It is advisable to refer to the publisher's version if you intend to cite from the work. See Guidance on citing.

To link to this article DOI: http://dx.doi.org/10.4064/sm228-2-1

Publisher: Institute of Mathematics Polish Academy of Sciences

All outputs in CentAUR are protected by Intellectual Property Rights law, including copyright law. Copyright and IPR is retained by the creators or other copyright holders. Terms and conditions for use of this material are defined in the End User Agreement.

www.reading.ac.uk/centaur 
Central Archive at the University of Reading

Reading's research outputs online 


\title{
FAILURE OF NEHARI'S THEOREM FOR MULTIPLICATIVE HANKEL FORMS IN SCHATTEN CLASSES
}

\author{
OLE FREDRIK BREVIG AND KARL-MIKAEL PERFEKT
}

\begin{abstract}
Ortega-Cerdà-Seip demonstrated that there are bounded multiplicative Hankel forms which do not arise from bounded symbols. On the other hand, when such a form is in the Hilbert-Schmidt class $\mathcal{S}_{2}$, Helson showed that it has a bounded symbol. The present work investigates forms belonging to the Schatten classes between these two cases. It is shown that for every $p>(1-\log \pi / \log 4)^{-1}$ there exist multiplicative Hankel forms in the Schatten class $\mathcal{S}_{p}$ which lack bounded symbols. The lower bound on $p$ is in a certain sense optimal when the symbol of the multiplicative Hankel form is a product of homogeneous linear polynomials.
\end{abstract}

\section{INTRODUCTION}

For a sequence $\varrho=\left(\varrho_{1}, \varrho_{2}, \varrho_{3}, \ldots\right) \in \ell^{2}$ its corresponding multiplicative Hankel form on $\ell^{2} \times \ell^{2}$ is given by

$$
\varrho(a, b)=\sum_{m=1}^{\infty} \sum_{n=1}^{\infty} \varrho_{m n} a_{m} b_{n},
$$

which initially is defined at least for finitely supported $a, b \in \ell^{2}$. Such forms are naturally understood as small Hankel operators on the Hardy space of the infinite polydisc, $H^{2}\left(\mathbb{D}^{\infty}\right)$. Therefore, one is led to investigate the relationship between the symbol - a function on the polytorus $\mathbb{T}^{\infty}$ generating the Hankel form - and the properties of the corresponding Hankel operator.

In the classical setting, (additive) Hankel forms are realized as Hankel operators on the Hardy space in the unit disc, $H^{2}(\mathbb{D})$. Nehari's theorem [8] states that every bounded Hankel form is generated by a bounded symbol on the torus $\mathbb{T}$.

On the infinite polydisc, the study of the corresponding statement was initiated by H. Helson [4, pp. 52-54], who raised the following questions.

Date: September 7, 2015.

2010 Mathematics Subject Classification. Primary 47B35. Secondary 30B50.

Key words and phrases. Hankel forms, infinite-dimensional torus, Schatten class, Nehari's theorem, Dirichlet series.

The first author is supported by Grant 227768 of the Research Council of Norway. 
Question 1. Does every bounded multiplicative Hankel form have a bounded symbol $\psi$ on the polytorus $\mathbb{T}^{\infty}$ ?

Question 2. Does every multiplicative Hankel form in the Hilbert-Schmidt class $\mathcal{S}_{2}$ have a bounded symbol?

Helson himself [5] gave a positive answer to Question 2. Ortega-Cerdà and Seip [9] proved that there are bounded multiplicative Hankel forms that do not have bounded symbols, using an idea of Helson [6], and hence gave a negative answer to Question 1. Furthermore, their argument also quickly produces that there are compact Hankel forms without bounded symbols (see Lemma 1). In light of these results, a next natural question to ask is:

Question 3. Does there exist a Hankel form belonging to a Schatten class $\mathcal{S}_{p}, 2<p<\infty$, without a bounded symbol? If so, for which values of $p$ does such a form exist?

We will answer the first part of this question, by showing that for every

$$
p>p_{0}=\left(1-\frac{\log \pi}{\log 4}\right)^{-1} \approx 5.738817179,
$$

there are multiplicative Hankel forms in $\mathcal{S}_{p}$ which do not have bounded symbols.

Our construction relies on independent products of homogeneous linear symbols and is optimal when testing against products of linear homogeneous polynomials, see Theorem 4. It is quite tempting to further conjecture that forms without bounded symbols can be found in $\mathcal{S}_{p}$ for every $p>2$, but our method does not substantiate this claim.

The paper is organized into two further sections. Section 2 reviews the connection between multiplicative Hankel forms, the Hardy space of Dirichlet series, and the Hardy space of the infinite polydisc. In Section 3 the main results are proven.

\section{Preliminaries}

We let $\mathscr{H}^{2}$ denote the Hilbert space of Dirichlet series

$$
f(s)=\sum_{n=1}^{\infty} a_{n} n^{-s}
$$

with square summable coefficients. If $g$ and $\varphi$ are Dirichlet series in $\mathscr{H}^{2}$ with coefficients $b_{n}$ and $\bar{\varrho}_{n}$, respectively, a computation shows that

$$
\langle f g, \varphi\rangle_{\mathscr{H}^{2}}=\varrho(a, b) .
$$


A key tool in the study of Hardy spaces of Dirichlet series is the Bohr lift [1]. For any $n \in \mathbb{N}$, the fundamental theorem of arithmetic yields the prime factorization

$$
n=\prod_{j=1}^{\infty} p_{j}^{\kappa_{j}},
$$

which associates the finite non-negative multi-index $\kappa(n)=\left(\kappa_{1}, \kappa_{2}, \kappa_{3}, \ldots\right)$ to $n$. The Bohr lift of the Dirichlet series (2) is the power series

$$
\mathscr{B} f(z)=\sum_{n=1}^{\infty} a_{n} z^{\kappa(n)},
$$

where $z=\left(z_{1}, z_{2}, z_{3}, \ldots\right)$. Hence (3) is a power series in countably infinite number of variables, but each term contains only a finite number of variables.

Under the Bohr lift, $\mathscr{H}^{2}$ corresponds to the infinite dimensional Hardy space $H^{2}\left(\mathbb{D}^{\infty}\right)$, which we view as a subspace of $L^{2}\left(\mathbb{T}^{\infty}\right)$. We refer to [3] for the details, mentioning only that the Haar measure of the compact abelian group $\mathbb{T}^{\infty}$ is simply the product of the normalized Lebesgue measures of each variable. In particular, $H^{2}\left(\mathbb{D}^{d}\right)$ is a natural subspace of $H^{2}\left(\mathbb{D}^{\infty}\right)$.

A formal computation shows that

$$
\langle\mathscr{B} f \mathscr{B} g, \mathscr{B} \varphi\rangle_{L^{2}\left(\mathbb{T}^{\infty}\right)}=\langle f g, \varphi\rangle_{\mathscr{H}^{2}},
$$

allowing us to compute the multiplicative Hankel form (1) on $\mathbb{T}^{\infty}$. In the remainder of this paper we work exclusively in the polydisc, with no reference to Dirichlet series. Therefore, we drop the notation $\mathscr{B}$ and study Hankel forms

$$
H_{\varphi}(f g)=\langle f g, \varphi\rangle_{L^{2}\left(\mathbb{T}^{\infty}\right)}, \quad f, g \in H^{2}\left(\mathbb{D}^{\infty}\right) .
$$

In the previous considerations we had that $\varphi \in H^{2}\left(\mathbb{D}^{\infty}\right)$, but there is nothing to prevent us from considering arbitrary symbols from $L^{2}\left(\mathbb{T}^{\infty}\right)$. Hence, each $\varphi \in L^{2}\left(\mathbb{T}^{\infty}\right)$ induces by (4) a (possibly unbounded) Hankel form $H_{\varphi}$ on $H^{2}\left(\mathbb{D}^{\infty}\right) \times H^{2}\left(\mathbb{D}^{\infty}\right)$. Of course, this is not a real generalization. Each form $H_{\varphi}$ is also induced by a symbol $\psi \in H^{2}\left(\mathbb{D}^{\infty}\right)$; letting $\psi=P \varphi$ we have $H_{\varphi}=H_{\psi}$, where $P$ denotes the orthogonal projection of $L^{2}\left(\mathbb{T}^{\infty}\right)$ onto $H^{2}\left(\mathbb{D}^{\infty}\right)$.

Note that if $\psi \in L^{\infty}\left(\mathbb{T}^{\infty}\right)$, then the corresponding multiplicative Hankel form is bounded, since

$$
\left|H_{\psi}(f g)\right|=|\langle f g, \psi\rangle| \leq\|f\|_{2}\|g\|_{2}\|\psi\|_{\infty} .
$$

We say that $H_{\varphi}$ has a bounded symbol if there exists a $\psi \in L^{\infty}\left(\mathbb{T}^{\infty}\right)$ such that $H_{\varphi}=H_{\psi}$. As mentioned in the introduction, it was shown in [9] that not every bounded multiplicative Hankel form has a bounded symbol. 
On the polydisc the Hankel form $H_{\varphi}$ is naturally realized as a (small) Hankel operator $\mathbf{H}_{\varphi}$, which when bounded acts as an operator from $H^{2}\left(\mathbb{D}^{\infty}\right)$ to the anti-analytic space $\overline{H^{2}}\left(\mathbb{D}^{\infty}\right)$. Letting $\bar{P}$ denote the orthogonal projection of $L^{2}\left(\mathbb{T}^{\infty}\right)$ onto $\overline{H^{2}}\left(\mathbb{D}^{\infty}\right)$, we have at least for polynomials $f \in H^{2}\left(\mathbb{D}^{\infty}\right)$ that

$$
\mathbf{H}_{\varphi} f=\bar{P}(\bar{\varphi} f)
$$

It is clear that when written in standard bases, the form $H_{\varphi}$ and the operator $\mathbf{H}_{\varphi}$ both correspond to the same infinite matrix

$$
M_{\varrho}=\left(\begin{array}{cccc}
\varrho_{1} & \varrho_{2} & \varrho_{3} & \cdots \\
\varrho_{2} & \varrho_{4} & \varrho_{6} & \cdots \\
\varrho_{3} & \varrho_{6} & \varrho_{9} & \cdots \\
\vdots & \vdots & \vdots & \ddots
\end{array}\right)
$$

Finally, we briefly recall the definition of the Schatten classes $\mathcal{S}_{p}, 0<p<\infty$. Assume that the Hankel form $H_{\varphi}$ is compact. Let $\Lambda=\left\{\lambda_{k}\right\}_{k=1}^{\infty}$ denote the singular value sequence of $M_{\varrho}$, which of course is the same as the singular value sequence of the operator $\mathbf{H}_{\varphi}$. The form $H_{\varphi}$, or equivalently the operator $\mathbf{H}_{\varphi}$, is in the Schatten class $\mathcal{S}_{p}$ if $\Lambda \in \ell^{p}$, and

$$
\left\|H_{\varphi}\right\|_{\mathcal{S}_{p}}=\left\|\mathbf{H}_{\varphi}\right\|_{\mathcal{S}_{p}}=\|\Lambda\|_{\ell^{p}}
$$

\section{Results}

To prove that there for each $p>p_{0}$ exist multiplicative Hankel forms in $\mathcal{S}_{p}$ without bounded symbols, we will assume that every $H_{\varphi} \in \mathcal{S}_{p}$ has a bounded symbol and derive a contradiction. We begin with the following routine lemma.

Lemma 1. Let $p \geq 1$. Assume that every $H_{\varphi} \in \mathcal{S}_{p}$ has a bounded symbol on $\mathbb{T}^{\infty}$. Then there is a constant $C_{p} \geq 1$ with the property that every $H_{\varphi} \in \mathcal{S}_{p}$ has a symbol $\psi \in L^{\infty}\left(\mathbb{T}^{\infty}\right)$ with $H_{\varphi}=H_{\psi}$ and such that $\|\psi\|_{\infty} \leq C_{p}\left\|H_{\varphi}\right\|_{\mathcal{S}_{p}}$.

Proof. We will define a lifting operator and show that it has to be continuous by appealing to the closed graph theorem.

Let $\mathrm{BH}$ denote the space of bounded multiplicative Hankel forms. By a standard argument it is isomorphic to the dual space of the weak product $\mathscr{H}^{2} \odot \mathscr{H}^{2}[6]$. In particular $\mathrm{BH}$ is a Banach space under the operator norm. It follows that $\mathcal{S}_{p} \mathrm{H}$ is also a Banach space, where $\mathcal{S}_{p} \mathrm{H}$ denotes the space of multiplicative Hankel forms in $\mathcal{S}_{p}$ equipped with the norm of $\mathcal{S}_{p}$. 
Now we define

$$
\begin{aligned}
& X=L^{\infty}\left(\mathbb{T}^{\infty}\right) \cap\left(L^{2}\left(\mathbb{T}^{\infty}\right) \ominus H^{2}\left(\mathbb{T}^{\infty}\right)\right), \\
& Y=L^{\infty}\left(\mathbb{T}^{\infty}\right) / X .
\end{aligned}
$$

$Y$ is a Banach space under the norm $\|\varphi\|_{Y}=\inf \left\{\|\psi\|_{\infty}: \psi-\varphi \in X\right\}$, seeing as $X$ is a closed subspace of $L^{\infty}\left(\mathbb{T}^{\infty}\right)$. Since by assumption every $H_{\varphi} \in \mathcal{S}_{p} \mathrm{H}$ has a symbol $\psi \in L^{\infty}\left(\mathbb{T}^{\infty}\right)$, we can define a map $T: \mathcal{S}_{p} \mathrm{H} \rightarrow Y$ by $T\left(H_{\varphi}\right)=\psi$. This is a well-defined linear map since $H_{\varphi}=0$ for a symbol $\varphi \in L^{\infty}\left(\mathbb{T}^{\infty}\right)$ if and only if $\varphi \in X$. An obvious computation verifies that $T$ is a closed operator, hence continuous. Therefore, there is a $C_{p} \geq 1$ such that

$$
\left\|T\left(H_{\varphi}\right)\right\|_{Y} \leq C_{p}\left\|H_{\varphi}\right\|_{\mathcal{S}_{p}}
$$

The statement of the lemma follows immediately.

Given the assumption of the lemma, we hence have for each polynomial $f$ and form $H_{\varphi} \in \mathcal{S}_{p}$ that

$|\langle f, \varphi\rangle|=\left|H_{\varphi}(f \cdot 1)\right|=\left|H_{\psi}(f \cdot 1)\right|=|\langle f, \psi\rangle| \leq\|\psi\|_{\infty}\|f\|_{1} \leq C_{p}\left\|H_{\varphi}\right\|_{\mathcal{S}_{p}}\|f\|_{1}$, where $\|\cdot\|_{1}$ denotes the norm of $L^{1}\left(\mathbb{T}^{\infty}\right)$. We thus obtain

$$
\frac{|\langle f, \varphi\rangle|}{\left\|H_{\varphi}\right\|_{\mathcal{S}_{p}}\|f\|_{1}} \leq C_{p}
$$

for every polynomial $f$ and every $H_{\varphi} \in \mathcal{S}_{p}$. To prove our main result we will construct a sequence of polynomials and finite rank forms to show that no finite constant $C_{p}$ satisfying (6) exists for $p>p_{0}$, thus obtaining a contradiction to the assumption of Lemma 1 . We will require the following lemma.

Lemma 2. Suppose that $\varphi_{1}, \varphi_{2}, \ldots, \varphi_{m}$ are symbols that depend on mutually separate variables and which generate the multiplicative Hankel forms $H_{\varphi_{j}} \in \mathcal{S}_{p}, 1 \leq j \leq m$. Then

$$
\left\|H_{\varphi}\right\|_{\mathcal{S}_{p}}=\left\|H_{\varphi_{1}}\right\|_{\mathcal{S}_{p}}\left\|H_{\varphi_{2}}\right\|_{\mathcal{S}_{p}} \cdots\left\|H_{\varphi_{m}}\right\|_{\mathcal{S}_{p}}
$$

where $\varphi=\varphi_{1} \varphi_{2} \cdots \varphi_{m}$.

Proof. For $1 \leq j \leq m$, we let $X_{j}$ denote the Hardy space of precisely the variables that the symbol $\varphi_{j}$ depends on, and if necessary let $X_{0}$ denote the Hardy space of the remaining variables, so that - as tensor products of Hilbert spaces — we have

$$
H^{2}\left(\mathbb{D}^{\infty}\right)=X_{0} \otimes X_{1} \otimes X_{2} \otimes \cdots X_{m}
$$


We set $\varphi_{0}=1$ and consider the small Hankel operators $\widetilde{\mathbf{H}}_{\varphi_{j}}: X_{j} \rightarrow \overline{X_{j}}$, defined similarly to (5) for $0 \leq j \leq m$. Now, if $f_{j} \in X_{j}, 0 \leq j \leq m$, we observe that

$$
\mathbf{H}_{\varphi}\left(f_{0} f_{1} \cdots f_{m}\right)=\widetilde{\mathbf{H}}_{\varphi_{0}}\left(f_{0}\right) \widetilde{\mathbf{H}}_{\varphi_{1}}\left(f_{1}\right) \cdots \widetilde{\mathbf{H}}_{\varphi_{m}}\left(f_{m}\right),
$$

and hence $\mathbf{H}_{\varphi}=\widetilde{\mathbf{H}}_{\varphi_{0}} \otimes \widetilde{\mathbf{H}}_{\varphi_{1}} \otimes \cdots \otimes \widetilde{\mathbf{H}}_{\varphi_{m}}$.

Note that $\widetilde{\mathbf{H}}_{\varphi_{0}}$ has the sole singular value 1, of multiplicity 1 . It follows that all singular values $\lambda$ of $\mathbf{H}_{\varphi}$ are obtained as products $\lambda=\lambda_{1} \lambda_{2} \cdots \lambda_{m}$, where $\lambda_{j}$ is a singular value of $\widetilde{\mathbf{H}}_{\varphi_{j}}$, see [2]. The multiplicity of $\lambda$ is also obtained in the expected way. From this, a short computation shows that

$$
\left\|\mathbf{H}_{\varphi}\right\|_{\mathcal{S}_{p}}=\left\|\widetilde{\mathbf{H}}_{\varphi_{1}}\right\|_{\mathcal{S}_{p}}\left\|\widetilde{\mathbf{H}}_{\varphi_{2}}\right\|_{\mathcal{S}_{p}} \cdots\left\|\widetilde{\mathbf{H}}_{\varphi_{m}}\right\|_{\mathcal{S}_{p}} .
$$

Finally, we have $\mathbf{H}_{\varphi_{j}}=\widetilde{\mathbf{H}}_{\varphi_{0}} \otimes \widetilde{\mathbf{H}}_{\varphi_{j}}$, where we now regard $\widetilde{\mathbf{H}}_{\varphi_{0}}$ as an operator on the Hardy space of the variables of which $\varphi_{j}$ is independent. Arguing as above, it follows that $\left\|\mathbf{H}_{\varphi_{j}}\right\|_{\mathcal{S}_{p}}=\left\|\widetilde{\mathbf{H}}_{\varphi_{j}}\right\|_{\mathcal{S}_{p}}$, completing the proof.

If $f_{1}, f_{2}, \ldots, f_{m}$ are polynomials depending on the same separate variables as $\varphi_{1}, \varphi_{2}, \ldots, \varphi_{m}$, respectively, and we set $f=f_{1} f_{2} \cdots f_{m}$, then

$$
\begin{aligned}
|\langle f, \varphi\rangle| & =\left|\left\langle f_{1}, \varphi_{1}\right\rangle\right|\left|\left\langle f_{2}, \varphi_{2}\right\rangle\right| \cdots\left|\left\langle f_{m}, \varphi_{m}\right\rangle\right|, \\
\|f\|_{1} & =\left\|f_{1}\right\|_{1}\left\|f_{2}\right\|_{1} \cdots\left\|f_{m}\right\|_{1} .
\end{aligned}
$$

Let $\mathrm{S}$ be the shift operator $\mathrm{S} f\left(z_{1}, z_{2}, \ldots\right)=f\left(z_{2}, z_{3}, \ldots\right)$. Suppose that we can find polynomials $f$ and $\varphi$, both depending on the first $d$ variables $z_{1}, z_{2}, \ldots, z_{d}$, satisfying

$$
\frac{|\langle f, \varphi\rangle|}{\left\|H_{\varphi}\right\|_{\mathcal{S}_{p}}\|f\|_{1}}>1
$$

Then, for $1 \leq j \leq m$, consider the functions

$$
\varphi_{j}(z)=\mathrm{S}^{d(j-1)} \varphi(z) \quad \text { and } \quad f_{j}(z)=\mathrm{S}^{d(j-1)} f(z) .
$$

With $\Phi=\varphi_{1} \varphi_{2} \cdots \varphi_{m}$ and $F=f_{1} f_{2} \cdots f_{m}$, Lemma 2 yields

$$
\frac{|\langle F, \Phi\rangle|}{\left\|H_{\Phi}\right\|_{\mathcal{S}_{p}}\|F\|_{1}}=\left(\frac{|\langle f, \varphi\rangle|}{\left\|H_{\varphi}\right\|_{\mathcal{S}_{p}}\|f\|_{1}}\right)^{m} \rightarrow \infty, \quad m \rightarrow \infty,
$$

giving us the sought contradiction to (6). We realize this scheme in the next theorem.

Theorem 3. For every $p>p_{0}$ there is a multiplicative Hankel form $H_{\varphi} \in \mathcal{S}_{p}$ which does not have a bounded symbol.

Proof. Let $d$ be a large positive integer to be chosen later. Consider the symbol

$$
\varphi(z)=\frac{z_{1}+z_{2}+z_{3}+\cdots+z_{d}}{\sqrt{d}}
$$


NEHARI'S THEOREM FOR MULTIPLICATIVE HANKEL FORMS IN $\mathcal{S}_{p}$

It is clear that the sequence $\varrho=\left(\varrho_{n}\right)_{n=1}^{\infty}$ for the matrix of $H_{\varphi}$ is given by

$$
\varrho_{n}= \begin{cases}1 / \sqrt{d} & \text { if } n=p_{j} \text { and } 1 \leq j \leq d \\ 0 & \text { otherwise }\end{cases}
$$

where $p_{j}$ denotes the $j$ th prime. In other terms, the matrix $M_{\varrho}$ of $H_{\varphi}$, with all zero rows and columns omitted, is the $(d+1) \times(d+1)$ matrix

$$
\frac{1}{\sqrt{d}}\left(\begin{array}{ccccc}
0 & 1 & 1 & \cdots & 1 \\
1 & 0 & 0 & \cdots & 0 \\
1 & 0 & 0 & \cdots & 0 \\
\vdots & \vdots & \vdots & \ddots & \vdots \\
1 & 0 & 0 & \cdots & 0
\end{array}\right) .
$$

This matrix is easily seen to have the singular values 1 (with multiplicity 2) and 0 (with multiplicity $d-1$ ), and thus

$$
\left\|H_{\varphi}\right\|_{\mathcal{S}_{p}}=2^{\frac{1}{p}}
$$

We choose $f(z)=\varphi(z)$. Then $\langle f, \varphi\rangle=1$, and, moreover, the central limit theorem for Steinhaus variables gives us that

$$
\lim _{d \rightarrow \infty}\|f\|_{1}=\lim _{d \rightarrow \infty} \mathbb{E}\left(\frac{\left|z_{1}+z_{2}+z_{3}+\cdots+z_{d}\right|}{\sqrt{d}}\right)=\frac{\sqrt{\pi}}{2} .
$$

In particular, for each $\delta>0$ we have for sufficiently large $d$ that

$$
\|f\|_{1} \leq \frac{\sqrt{\pi}}{2}+\delta
$$

We now observe that $p=p_{0}$ is the solution of the equation $2^{1 / p} \cdot \sqrt{\pi} / 2=1$, and hence if $p>p_{0}$ we may find $\delta>0$ small enough that

$$
\left\|H_{\varphi}\right\|_{\mathcal{S}_{p}} \cdot\|f\|_{1} \leq 2^{1 / p} \cdot\left(\frac{\sqrt{\pi}}{2}+\delta\right)<1 .
$$

This implies that if $d$ is large enough, $f$ and $\varphi$ satisfy (8). This completes the proof by appealing to the discussion preceding the statement of the theorem.

Our result is optimal for symbols which are independent products of linear homogeneous polynomials and test functions of the same form, as shown by the following result.

Theorem 4. Suppose $p \leq p_{0}$ and consider

$$
\begin{aligned}
& \qquad(z)=a_{1} z_{1}+a_{2} z_{2}+\cdots+a_{d} z_{d} \quad \text { and } \quad f(z)=b_{1} z_{1}+b_{2} z_{2}+\cdots+b_{d} z_{d}, \\
& \text { for } a_{j}, b_{j} \in \mathbb{C} \text {. Then }|\langle f, \varphi\rangle| \leq\left\|H_{\varphi}\right\|_{\mathcal{S}_{p}}\|f\|_{1} \text {. }
\end{aligned}
$$


Proof. By the Cauchy-Schwarz inequality and Parseval's formula, it is clear that

$$
|\langle f, \varphi\rangle| \leq\|a\|_{\ell^{2}}\|b\|_{\ell^{2}}
$$

Straightforward computations with the matrix $M_{\varrho}$ of $H_{\varphi}$ show that

$$
M_{\varrho} M_{\varrho}^{*}=\left(\begin{array}{ccccc}
\|a\|_{\ell^{2}}^{2} & 0 & 0 & \cdots & 0 \\
0 & a_{1} \overline{a_{1}} & a_{1} \overline{a_{2}} & \cdots & a_{1} \overline{a_{d}} \\
0 & a_{2} \overline{a_{1}} & a_{2} \overline{a_{2}} & \cdots & a_{2} \overline{a_{d}} \\
\vdots & \vdots & \vdots & \ddots & \vdots \\
0 & a_{d} \overline{a_{1}} & a_{d} \overline{a_{2}} & \cdots & a_{d} \overline{a_{d}}
\end{array}\right) .
$$

Here we have again omitted zero rows and columns. Note that the lower right block has rank 1 . By considering the vector $\left(0, a_{1}, a_{2}, \ldots, a_{d}\right)$ it is clear that it has the sole eigenvalue $\|a\|_{\ell^{2}}^{2}$. Thus, the singular value sequence of $M_{\varrho}$ is $\Lambda=\left\{\|a\|_{\ell^{2}},\|a\|_{\ell^{2}}, 0, \ldots, 0\right\}$, and hence

$$
\left\|H_{\varphi}\right\|_{\mathcal{S}_{p}}=2^{1 / p}\|a\|_{\ell^{2}}
$$

We use the optimal Khintchine inequality for Steinhaus variables [7, 10], $p=1$, and obtain

$$
\|f\|_{1} \geq \frac{\sqrt{\pi}}{2}\|b\|_{\ell^{2}}
$$

The hypothesis that $p \leq p_{0}$ implies that $2^{1 / p} \sqrt{\pi} / 2 \geq 1$, and the proof is finished by the following chain of inequalities.

$$
\left\|H_{\varphi}\right\|_{\mathcal{S}_{p}} \cdot\|f\|_{1} \geq 2^{1 / p} \cdot\|a\|_{\ell^{2}} \cdot \frac{\sqrt{\pi}}{2} \cdot\|b\|_{\ell^{2}} \geq\|a\|_{\ell^{2}} \cdot\|b\|_{\ell^{2}} \geq|\langle f, \varphi\rangle| .
$$

\section{REFERENCES}

1. H. Bohr, Ueber die Bedeutung der Potenzreihen unendlich vieler Variablen in der Theorie der Dirichletschen Reihe, Nachr. Akad. Wiss. Göttingen Math.-Phys. 1913 (1913), 441-488.

2. A. Brown and C. Pearcy, Spectra of tensor products of operators, Proc. Amer. Math. Soc. 17 (1966), no. 1, 162-166.

3. H. Hedenmalm, P. Lindqvist, and K. Seip, A Hilbert space of Dirichlet series and systems of dilated functions in $L^{2}(0,1)$, Duke Math. J. 86 (1997), no. 1, 1-37.

4. H. Helson, Dirichlet series, Henry Helson, 2005.

5. _ Hankel forms and sums of random variables, Studia Math. 176 (2006), 8592.

6. _ Hankel forms, Studia Math. 198 (2010), 79-84.

7. H. König, On the best constants in the Khintchine inequality for Steinhaus variables, Israel J. Math. (2013), 1-35.

8. Z. Nehari, On bounded bilinear forms, Ann. of Math. (1957), 153-162.

9. J. Ortega-Cerdà and K. Seip, A lower bound in Nehari's theorem on the polydisc, J. Anal. Math. 118 (2012), no. 1, 339-342.

10. J. Sawa, The best constant in the Khintchine inequality for complex Steinhaus variables, the case $p=1$, Studia Math. 81 (1985), no. 1, 107-126. 
NEHARI'S THEOREM FOR MULTIPLICATIVE HANKEL FORMS IN $\mathcal{S}_{p}$

Department of Mathematical Sciences, Norwegian University of Science and Technology (NTNU), NO-7491 Trondheim, Norway

E-mail address: ole.brevig@math.ntnu.no

Department of Mathematical Sciences, Norwegian University of Science and Technology (NTNU), NO-7491 Trondheim, Norway

E-mail address: karl-mikael.perfekt@math.ntnu.no 